CRYSTALLOGRAPHIC COMMUNICATIONS

ISSN 2056-9890

Received 30 April 2015

Accepted 13 May 2015

Edited by M. Weil, Vienna University of Technology, Austria

Keywords: crystal structure; 4-(trimethylgermyl)benzoic acid; germanium; hydrogen bonding

CCDC reference: 1400647

Supporting information: this article has supporting information at journals.iucr.org/e

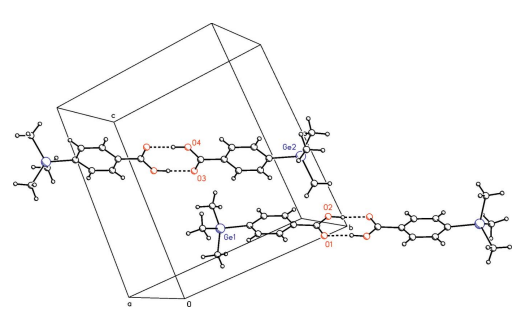

OPEN $\odot$ ACCESS

\section{Crystal structure of 4-(trimethylgermyl)benzoic acid}

\author{
Lena Knauer, Eva R. Barth, Christopher Golz and Carsten Strohmann*
}

Fakultät für Chemie und Chemische Biologie, Technische Universität Dortmund, Otto-Hahn-Strasse 6, 44227 Dortmund, Germany. *Correspondence e-mail: carsten.strohmann@tu-dortmund.de

The title compound, $\left[\mathrm{Ge}\left(\mathrm{CH}_{3}\right)_{3}\left(\mathrm{C}_{7} \mathrm{H}_{5} \mathrm{O}_{2}\right)\right]$, was obtained as a by-product in the synthesis of the corresponding aldehyde. Two slightly different molecules are present in the asymmetric unit. In both molecules, the geometry of the aromatic ring plane is distorted by varying intensities. Additionally, the Ge atoms deviate from the mean aromatic ring planes. Whereas the distance of the $\mathrm{Ge}$ atom to the ring plane is only 0.101 (4) $\AA$ in the first molecule, this distance is increased to 0.210 (4) $\AA$ in the second. In the crystal structure, centrosymmetric $\mathrm{O}-\mathrm{H} \cdots \mathrm{O}$ hydrogen-bonded dimers are formed. The title compound is isostructural with the Si analogue [Haberecht et al. (2004). Acta Cryst. E60, o329-0330].

\section{Chemical context}

The application of 1,4-dihydropyridines (DHPs) as a pharmaceutical tool represents a novel and promising approach in the therapy of autoimmune diseases, cancer and other illnesses. The effect of drugs containing DHPs is based on the interaction with the Transforming Growth Factor $\beta$ (TGF $\beta$ ). The title compound, $\left[\mathrm{Ge}\left(\mathrm{CH}_{3}\right)_{3}\left(\mathrm{C}_{7} \mathrm{H}_{5} \mathrm{O}_{2}\right)\right]$, (I), was obtained as a side-product in the synthesis of the corresponding aldehyde, which can be employed in the synthesis of DHPs (Längle et al., 2015).<smiles>C[Ge](C)(C)c1ccc(C(=O)O)cc1</smiles>

\section{Structural commentary}

The asymmetric unit of (I) contains two molecules (Fig. 1), which exhibit different deformations of the aromatic plane. This deformation may be caused by the sterically demanding substituents in 1- and 4-positions. In the first molecule, the opposite carbon atoms $\mathrm{C} 2$ and $\mathrm{C} 5$ deviate from the mean aromatic ring plane by -0.015 (2) $\AA$, which leads to a boatshaped deformation (Table 1). The distance of the germanium atom Ge1 to this plane is -0.210 (4) $\AA$. Corresponding to this boat-shaped deformation, the bond lengths of the aromatic ring are not equidistant, but can be divided into three pairs of similar distances: the bonds C5-C4 [1.393 (4) $\mathrm{A}]$ and C5-C6

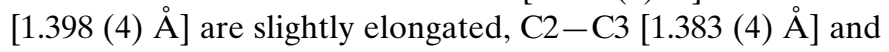


Table 1

Deviation of atoms from the benzene ring least-squares planes $(\AA)$.

\begin{tabular}{lrlr}
\hline Atom & \multicolumn{1}{l}{ Deviation } & Atom & \multicolumn{1}{c}{ Deviation } \\
\hline C2 & $-0.015(2)$ & $\mathrm{C} 12$ & $0.004(2)$ \\
C3 & $0.007(2)$ & $\mathrm{C} 13$ & $-0.003(2)$ \\
C4 & $0.008(2)$ & $\mathrm{C} 14$ & $-0.003(2)$ \\
C5 & $-0.015(2)$ & $\mathrm{C} 15$ & $0.007(2)$ \\
C6 & $0.007(2)$ & $\mathrm{C} 16$ & $-0.006(2)$ \\
C7 & $0.007(2)$ & $\mathrm{C} 17$ & $0.000(4)$ \\
Ge1* & $-0.210(4)$ & Ge2* & $0.101(4)$ \\
\hline
\end{tabular}

Note: $(*)$ not used in the least-squares-plane calculation

C2-C7 [1.384 (4) $\AA$ ] lie in a medium range, and C3-C4 [1.368 (4) $\AA]$ and C6-C7 [1.379 (4) $\AA]$ are the shortest. In the second molecule, the aromatic ring exhibits a nearly planar geometry (Table 1). Similar to the first molecule, the Ge2 atom deviates from the mean aromatic ring plane by 0.101 (4) $\AA$. Additionally, elongated bond lengths at $\mathrm{C} 12$ and $\mathrm{C} 15$ can be observed [C12-C13 1.385 (4), C12-C17 1.381 (4), C15-C14 1.393 (4), C15-C16 1.398 (4) Å].

All in all, the degree of deformation in the second molecule is smaller compared to the first molecule. This difference may be the reason for the presence of two molecules in the asymmetric unit. The deformations described above may be caused by the sterically demanding substituents attached to the aromatic ring in 1- and 4-positions, or may be traced back to packing effects.

\section{Supramolecular features}

The molecules in the title compound crystallize as centrosymmetric hydrogen-bonded dimers (Fig. 2, Table 2). Considering the donor $\cdots$ acceptor bond lengths of 2.626 (3) $\AA$ $[\mathrm{O} 2-\mathrm{H} 2 \cdots \mathrm{O} 1]$ and $2.635(3) \AA[\mathrm{O} 4-\mathrm{H} 4 A \cdots \mathrm{O} 3]$, the strength of the hydrogen bonds can be classified as moderate according to Jeffrey (1997).

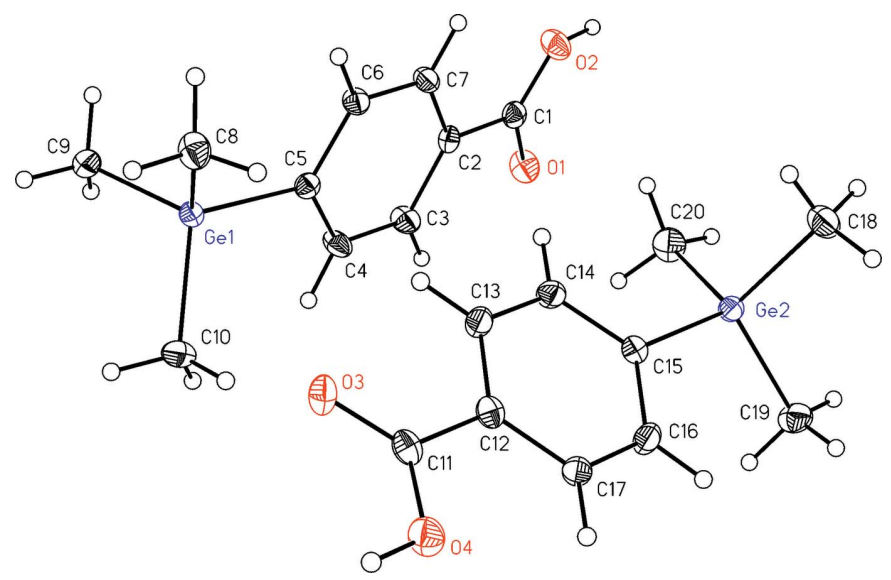

Figure 1

The structures and atom numbering of the two independent molecules in the title compound. Displacement ellipsoids are drawn at the $30 \%$ probability level.
Table 2

Hydrogen-bond geometry $\left(\AA,{ }^{\circ}\right)$.

\begin{tabular}{lllll}
\hline$D-\mathrm{H} \cdots A$ & $D-\mathrm{H}$ & $\mathrm{H} \cdots A$ & $D \cdots A$ & $D-\mathrm{H} \cdots A$ \\
\hline $\mathrm{O} 2-\mathrm{H} 2 \cdots \mathrm{O} 1^{\mathrm{i}}$ & $0.93(5)$ & $1.71(5)$ & $2.626(3)$ & $170(5)$ \\
$\mathrm{O} 4-\mathrm{H} 4 A \cdots 3^{\mathrm{ii}}$ & $0.93(5)$ & $1.70(5)$ & $2.635(3)$ & $179(4)$ \\
\hline
\end{tabular}

Symmetry codes: (i) $-x,-y+2,-z$; (ii) $-x+2,-y+1,-z+1$.

\section{Database survey}

In the isotypic structure containing silicon instead of germanium, similar distortions can be observed (Haberecht et al., 2004). In this structure, the asymmetric unit also contains two differently deformed molecules. In the first molecule, a nearly planar geometry of the aromatic ring plane is exhibited. The second molecule shows the same boat-shaped deformation of the aromatic ring as described for the Ge compound. The atoms equal to $\mathrm{C} 12$ and $\mathrm{C} 15$ deviate by -0.016 (1) $\AA$ and -0.017 (1) $\AA$, respectively. The silicon atoms Si1 and Si2 exhibit distances to the aromatic ring plane of 0.088 (3) and -0.219 (2) $\AA$, respectively. A comparison of these distances to those reported for the title compound reveals that the observed distortions occur in similar dimensions for both structures. This points to a comparable steric demand of the trimethylgermyl and trimethylsilyl moieties.

\section{Synthesis and crystallization}

To a solution of 1,4-dibromobenzene $(1.50 \mathrm{~g}, 6.36 \mathrm{mmol})$ in $\mathrm{Et}_{2} \mathrm{O}(13 \mathrm{ml})$ was added $n$-BuLi (6.36 mmol, $2.5 \mathrm{M}$ in hexane) at $195 \mathrm{~K}$ and the mixture stirred at this temperature for $4 \mathrm{~h}$. Then chlorotrimethylgermane $(1.10 \mathrm{~g}, 7.00 \mathrm{mmol})$ was added to the reaction mixture at $195 \mathrm{~K}$, stirred at this temperature for $10 \mathrm{~min}$, followed by stirring over night at room temperature. After addition of $\mathrm{H}_{2} \mathrm{O}$, the organic phase was separated and the aqueous phase was extracted with $\mathrm{Et}_{2} \mathrm{O}$ three times. The combined organic phases were washed with brine and dried over $\mathrm{Na}_{2} \mathrm{SO}_{4}$. Removal of the solvent under reduced pressure afforded (4-bromophenyl)trimethylgermane (1.67 g,

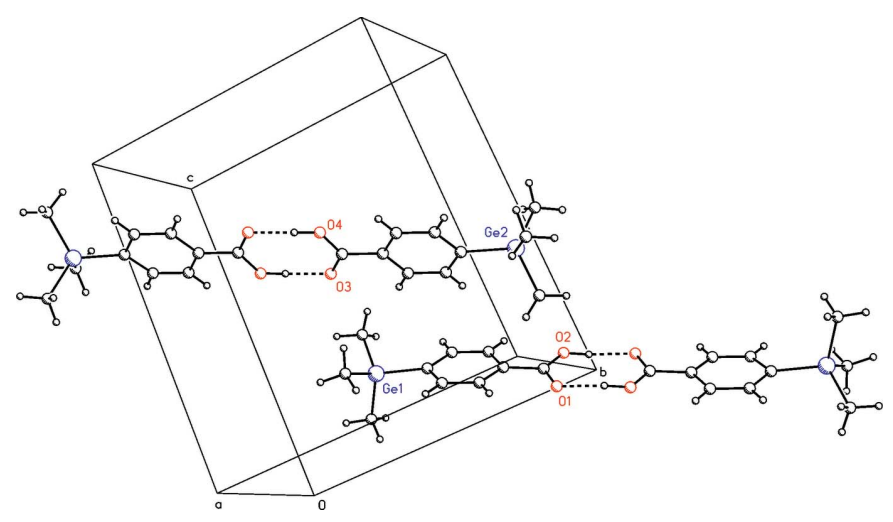

Figure 2

Illustration of the hydrogen-bonded dimers in the unit cell. Hydrogen bonds are represented as dashed lines. 


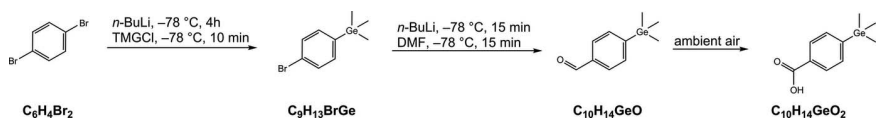

Figure 3

Schematic representation of the synthesis of compound (I).

$6.12 \mathrm{mmol}, 96 \%$ ) as a colorless liquid. The reaction product was used in following syntheses without further purification.

To a solution of (4-bromophenyl)trimethylgermane $(1.67 \mathrm{~g}$, $6.12 \mathrm{mmol})$ in THF $(38 \mathrm{ml})$ was added $n$-BuLi $(6.73 \mathrm{mmol}, 2.5$ $M$ in hexane) at $195 \mathrm{~K}$ and the mixture was stirred at this temperature for 15 minutes. Then dimethylformamide $(1.34 \mathrm{~g}$, $18.4 \mathrm{mmol}$ ) was added to the reaction mixture at $195 \mathrm{~K}$, and it was allowed to warm to room temperature over night. After addition of a saturated aqueous $\mathrm{NH}_{4} \mathrm{Cl}$ solution, the organic phase was separated and the aqueous phase extracted three times with $\mathrm{Et}_{2} \mathrm{O}$. The combined organic phases were washed with water and brine and dried over $\mathrm{Na}_{2} \mathrm{SO}_{4}$. Removal of the solvent under reduced pressure and subsequent silica gel chromatography (pentane, pentane $/ \mathrm{Et}_{2} \mathrm{O}=100: 1 \rightarrow 50: 1$ ) afforded 4-(trimethylgermyl)benzaldehyde, which oxidized at ambient air conditions to give 4-(trimethylgermyl)benzoic acid, (I), (1.05 g, $4.70 \mathrm{mmol}, 77 \%$ ) as a colorless solid. A schematic representation of the synthetic procedure is shown in Fig. 3.

\section{Refinement}

Crystal data, data collection and structure refinement details are summarized in Table 3. Hydrogen atoms were located from difference Fourier maps. They were refined with idealized positions in a riding model with $U_{\text {iso }}(\mathrm{H})=1.2 U_{\text {eq }}(\mathrm{C})$ and $\mathrm{C}-\mathrm{H}=0.95 \AA$ for aromatic hydrogen atoms, and with $U_{\text {iso }}(\mathrm{H})$ $=1.5 U_{\text {eq }}(\mathrm{C})$ and $\mathrm{C}-\mathrm{H}=0.98 \AA$ for methyl hydrogen atoms. All $\mathrm{CH}_{3}$ hydrogen atoms were allowed to rotate but not to tip. Hydroxyl hydrogen atoms were located from difference Fourier maps and were refined freely.

\section{Acknowledgements}

We are grateful to the Deutsche Forschungsgemeinschaft (DFG) for financial support.
Table 3

Experimental details.

\begin{tabular}{|c|c|}
\hline \multicolumn{2}{|l|}{ Crystal data } \\
\hline Chemical formula & {$\left[\mathrm{Ge}\left(\mathrm{CH}_{3}\right)_{3}\left(\mathrm{C}_{7} \mathrm{H}_{5} \mathrm{O}_{2}\right)\right]$} \\
\hline$M_{\mathrm{r}}$ & 238.80 \\
\hline Crystal system, space group & Triclinic, $P \overline{1}$ \\
\hline Temperature (K) & 173 \\
\hline$a, b, c(\AA)$ & $6.3560(4), 12.3927(6), 14.2084(7)$ \\
\hline$\alpha, \beta, \gamma\left({ }^{\circ}\right)$ & $96.348(4), 92.846(4), 93.246(4)$ \\
\hline$V\left(\AA^{3}\right)$ & $1108.76(10)$ \\
\hline$Z$ & 4 \\
\hline Radiation type & Мo $K \alpha$ \\
\hline$\mu\left(\mathrm{mm}^{-1}\right)$ & 2.73 \\
\hline Crystal size $(\mathrm{mm})$ & $0.08 \times 0.08 \times 0.02$ \\
\hline \multicolumn{2}{|l|}{ Data collection } \\
\hline Diffractometer & Agilent Xcalibur Sapphire3 \\
\hline Absorption correction & $\begin{array}{l}\text { Multi-scan (CrysAlis PRO; } \\
\text { Agilent, 2014) }\end{array}$ \\
\hline$T_{\min }, T_{\max }$ & $0.794,1.000$ \\
\hline $\begin{array}{l}\text { No. of measured, independent and } \\
\text { observed }[I>2 \sigma(I)] \text { reflections }\end{array}$ & $15667,4781,3261$ \\
\hline$R_{\text {int }}$ & 0.044 \\
\hline$(\sin \theta / \lambda)_{\max }\left(\AA^{-1}\right)$ & 0.639 \\
\hline \multicolumn{2}{|l|}{ Refinement } \\
\hline$R\left[F^{2}>2 \sigma\left(F^{2}\right)\right], w R\left(F^{2}\right), S$ & $0.036,0.083,1.02$ \\
\hline No. of reflections & 4781 \\
\hline No. of parameters & 249 \\
\hline $\mathrm{H}$-atom treatment & $\begin{array}{l}\mathrm{H} \text { atoms treated by a mixture of } \\
\text { independent and constrained } \\
\text { refinement }\end{array}$ \\
\hline$\Delta \rho_{\max }, \Delta \rho_{\min }\left(\mathrm{e} \AA^{-3}\right)$ & $0.54,-0.31$ \\
\hline
\end{tabular}

Computer programs: CrysAlis PRO (Agilent, 2014), SHELXS97 (Sheldrick, 2008), SHELXL2014 (Sheldrick, 2015) and OLEX2 (Dolomanov et al., 2009).

\section{References}

Agilent (2014). CrysAlis PRO. Agilent Technologies UK Ltd, Yarnton, England.

Dolomanov, O. V., Bourhis, L. J., Gildea, R. J., Howard, J. A. K. \& Puschmann, H. (2009). J. Appl. Cryst. 42, 339-341.

Haberecht, M. C., Vitze, H., Lerner, H.-W. \& Bolte, M. (2004). Acta Cryst. E60, o329-o330.

Jeffrey, G. A. (1997). In An Introduction to Hydrogen Bonding. Oxford University Press.

Längle, D., Marquardt, V., Heider, E., Vigante, B., Duburs, G., Luntena, I., Flötgen, D., Golz, C., Strohmann, C., Koch, O. \& Schade, D. (2015). Eur. J. Med. Chem. 95, 249-266.

Sheldrick, G. M. (2008). Acta Cryst. A64, 112-122.

Sheldrick, G. M. (2015). Acta Cryst. C71, 3-8. 


\section{supporting information}

Acta Cryst. (2015). E71, 687-689 [doi:10.1107/S2056989015009160]

\section{Crystal structure of 4-(trimethylgermyl)benzoic acid}

\section{Lena Knauer, Eva R. Barth, Christopher Golz and Carsten Strohmann}

\section{Computing details}

Data collection: CrysAlis PRO (Agilent, 2014); cell refinement: CrysAlis PRO (Agilent, 2014); data reduction: CrysAlis PRO (Agilent, 2014); program(s) used to solve structure: SHELXS97 (Sheldrick, 2008); program(s) used to refine structure: SHELXL2014 (Sheldrick, 2015); software used to prepare material for publication: OLEX2 (Dolomanov et al., 2009).

\section{4-(Trimethylgermyl)benzoic acid}

Crystal data

$\left[\mathrm{Ge}\left(\mathrm{CH}_{3}\right)_{3}\left(\mathrm{C}_{7} \mathrm{H}_{5} \mathrm{O}_{2}\right)\right]$

$Z=4$

$M_{r}=238.80$

Triclinic, $P \overline{1}$

$a=6.3560(4) \AA$

$b=12.3927$ (6) $\AA$

$c=14.2084(7) \AA$

$F(000)=488$

$D_{\mathrm{x}}=1.431 \mathrm{Mg} \mathrm{m}^{-3}$

Mo $K \alpha$ radiation, $\lambda=0.71073 \AA$

$\alpha=96.348(4)^{\circ}$

$\beta=92.846(4)^{\circ}$

Cell parameters from 4611 reflections

$\theta=2.9-28.4^{\circ}$

$\mu=2.73 \mathrm{~mm}^{-1}$

$\gamma=93.246(4)^{\circ}$

$T=173 \mathrm{~K}$

$V=1108.76(10) \AA^{3}$

Plate, clear colourless

$0.08 \times 0.08 \times 0.02 \mathrm{~mm}$

\section{Data collection}

Agilent Xcalibur Sapphire3 diffractometer

Radiation source: Enhance (Mo) X-ray Source

Graphite monochromator

Detector resolution: 16.0560 pixels $\mathrm{mm}^{-1}$

$\omega$ scans

Absorption correction: multi-scan

(CrysAlis PRO; Agilent, 2014)

$T_{\min }=0.794, T_{\max }=1.000$

15667 measured reflections

4781 independent reflections

3261 reflections with $I>2 \sigma(I)$

$R_{\text {int }}=0.044$

$\theta_{\max }=27.0^{\circ}, \theta_{\min }=2.3^{\circ}$

$h=-8 \rightarrow 8$

$k=-15 \rightarrow 15$

$l=-18 \rightarrow 18$

Refinement

Refinement on $F^{2}$

Least-squares matrix: full

$R\left[F^{2}>2 \sigma\left(F^{2}\right)\right]=0.036$

$w R\left(F^{2}\right)=0.083$

$S=1.01$

4781 reflections

249 parameters

0 restraints

Primary atom site location: structure-invariant direct methods

Hydrogen site location: mixed

$\mathrm{H}$ atoms treated by a mixture of independent and constrained refinement

$w=1 /\left[\sigma^{2}\left(F_{\mathrm{o}}^{2}\right)+(0.0332 P)^{2}\right]$ where $P=\left(F_{\mathrm{o}}^{2}+2 F_{\mathrm{c}}^{2}\right) / 3$

$(\Delta / \sigma)_{\max }<0.001$

$\Delta \rho_{\max }=0.54$ e $\AA^{-3}$

$\Delta \rho_{\min }=-0.31{\mathrm{e} \AA^{-3}}^{-3}$ 


\section{Special details}

Geometry. All e.s.d.'s (except the e.s.d. in the dihedral angle between two 1.s. planes) are estimated using the full covariance matrix. The cell e.s.d.'s are taken into account individually in the estimation of e.s.d.'s in distances, angles and torsion angles; correlations between e.s.d.'s in cell parameters are only used when they are defined by crystal symmetry. An approximate (isotropic) treatment of cell e.s.d.'s is used for estimating e.s.d.'s involving l.s. planes.

Fractional atomic coordinates and isotropic or equivalent isotropic displacement parameters $\left(\AA^{2}\right)$

\begin{tabular}{|c|c|c|c|c|}
\hline & $x$ & $y$ & $z$ & $U_{\text {iso }} * / U_{\text {eq }}$ \\
\hline Ge1 & $0.34904(5)$ & $0.43946(2)$ & $0.19617(2)$ & $0.02258(10)$ \\
\hline $\mathrm{O} 1$ & $0.1858(3)$ & $0.90478(18)$ & $-0.01761(15)$ & $0.0386(6)$ \\
\hline $\mathrm{O} 2$ & $-0.0693(4)$ & $0.9178(2)$ & $0.08546(16)$ & $0.0369(6)$ \\
\hline $\mathrm{H} 2$ & $-0.124(7)$ & $0.976(4)$ & $0.060(3)$ & $0.13(2)^{*}$ \\
\hline $\mathrm{C} 1$ & $0.0846(5)$ & $0.8712(3)$ & $0.0511(2)$ & $0.0268(7)$ \\
\hline $\mathrm{C} 2$ & $0.1569(5)$ & $0.7738(2)$ & $0.0910(2)$ & $0.0237(7)$ \\
\hline $\mathrm{C} 3$ & $0.3337(5)$ & $0.7244(3)$ & $0.0588(2)$ & $0.0309(8)$ \\
\hline H3 & 0.4140 & 0.7559 & 0.0131 & $0.037^{*}$ \\
\hline $\mathrm{C} 4$ & $0.3942(5)$ & $0.6307(3)$ & $0.0920(2)$ & $0.0306(8)$ \\
\hline H4 & 0.5164 & 0.5985 & 0.0688 & $0.037 *$ \\
\hline $\mathrm{C} 5$ & $0.2814(4)$ & $0.5813(2)$ & $0.1589(2)$ & $0.0230(7)$ \\
\hline C6 & $0.1072(4)$ & $0.6339(2)$ & $0.1931(2)$ & $0.0259(7)$ \\
\hline H6 & 0.0291 & 0.6039 & 0.2404 & $0.031^{*}$ \\
\hline C7 & $0.0460(5)$ & $0.7283(2)$ & $0.1598(2)$ & $0.0262(7)$ \\
\hline H7 & -0.0732 & 0.7624 & 0.1843 & $0.031 *$ \\
\hline $\mathrm{C} 8$ & $0.2619(5)$ & $0.4263(3)$ & $0.3237(2)$ & $0.0355(8)$ \\
\hline H8A & 0.3064 & 0.3576 & 0.3438 & $0.053^{*}$ \\
\hline H8B & 0.1079 & 0.4275 & 0.3244 & $0.053^{*}$ \\
\hline $\mathrm{H} 8 \mathrm{C}$ & 0.3273 & 0.4872 & 0.3672 & $0.053 *$ \\
\hline C9 & $0.1964(5)$ & $0.3288(3)$ & $0.1066(2)$ & $0.0324(8)$ \\
\hline H9A & 0.2346 & 0.3388 & 0.0421 & $0.049^{*}$ \\
\hline H9B & 0.0442 & 0.3351 & 0.1115 & $0.049^{*}$ \\
\hline $\mathrm{H} 9 \mathrm{C}$ & 0.2334 & 0.2565 & 0.1213 & $0.049^{*}$ \\
\hline $\mathrm{C} 10$ & $0.6506(4)$ & 0.4247 (3) & $0.1914(2)$ & $0.0361(8)$ \\
\hline $\mathrm{H} 10 \mathrm{~A}$ & 0.7241 & 0.4701 & 0.2453 & $0.054^{*}$ \\
\hline H10B & 0.6991 & 0.4480 & 0.1320 & $0.054^{*}$ \\
\hline $\mathrm{H} 10 \mathrm{C}$ & 0.6808 & 0.3484 & 0.1945 & $0.054^{*}$ \\
\hline $\mathrm{Ge} 2$ & $0.64145(5)$ & $1.05828(3)$ & $0.30054(2)$ & $0.02464(10)$ \\
\hline $\mathrm{O} 3$ & $0.7978(3)$ & $0.54301(17)$ & $0.43295(15)$ & $0.0338(5)$ \\
\hline $\mathrm{O} 4$ & $1.0933(4)$ & $0.6355(2)$ & $0.49737(16)$ & $0.0376(6)$ \\
\hline $\mathrm{H} 4 \mathrm{~A}$ & $1.133(7)$ & $0.573(4)$ & $0.522(3)$ & $0.116(19)^{*}$ \\
\hline $\mathrm{C} 11$ & $0.9143(5)$ & $0.6285(3)$ & $0.4488(2)$ & 0.0267 (7) \\
\hline C12 & $0.8508(5)$ & $0.7298(3)$ & $0.4112(2)$ & $0.0250(7)$ \\
\hline $\mathrm{C} 13$ & $0.6552(5)$ & $0.7320(2)$ & $0.3641(2)$ & $0.0276(7)$ \\
\hline H13 & 0.5620 & 0.6686 & 0.3551 & $0.033^{*}$ \\
\hline $\mathrm{C} 14$ & $0.5962(5)$ & $0.8266(2)$ & $0.3304(2)$ & $0.0275(7)$ \\
\hline H14 & 0.4618 & 0.8271 & 0.2981 & $0.033^{*}$ \\
\hline $\mathrm{C} 15$ & $0.7279(5)$ & $0.9214(2)$ & $0.3421(2)$ & $0.0244(7)$ \\
\hline C16 & $0.9254(5)$ & $0.9163(3)$ & $0.3887(2)$ & $0.0306(8)$ \\
\hline
\end{tabular}




$\begin{array}{lllll}\text { H16 } & 1.0205 & 0.9790 & 0.3968 & 0.037^{*} \\ \text { C17 } & 0.9849(5) & 0.8226(2) & 0.4229(2) & 0.0278(7) \\ \text { H17 } & 1.1195 & 0.8215 & 0.4549 & 0.033^{*} \\ \text { C18 } & 0.4858(5) & 1.1327(3) & 0.4005(2) & 0.0401(9) \\ \text { H18A } & 0.3884 & 1.0801 & 0.4254 & 0.060^{*} \\ \text { H18B } & 0.4055 & 1.1890 & 0.3746 & 0.060^{*} \\ \text { H18C } & 0.5851 & 1.1665 & 0.4517 & 0.060^{*} \\ \text { C19 } & 0.8925(5) & 1.1465(3) & 0.2791(2) & 0.0383(9) \\ \text { H19A } & 0.9715 & 1.1690 & 0.3398 & 0.057^{*} \\ \text { H19B } & 0.8517 & 1.2110 & 0.2503 & 0.057^{*} \\ \text { H19C } & 0.9813 & 1.1039 & 0.2365 & 0.057^{*} \\ \text { C20 } & 0.4583(5) & 1.0264(3) & 0.1866(2) & 0.0404(9) \\ \text { H20A } & 0.5249 & 0.9760 & 0.1408 & 0.061^{*} \\ \text { H20B } & 0.4346 & 1.0940 & 0.1588 & 0.061^{*} \\ \text { H20C } & 0.3229 & 0.9932 & 0.2024 & 0.061^{*}\end{array}$

Atomic displacement parameters $\left(\AA^{2}\right)$

\begin{tabular}{lllllll}
\hline & $U^{11}$ & $U^{22}$ & $U^{33}$ & $U^{12}$ & $U^{13}$ & $U^{23}$ \\
\hline Ge1 & $0.02235(19)$ & $0.02012(19)$ & $0.02573(19)$ & $0.00270(14)$ & $0.00329(14)$ & $0.00304(14)$ \\
O1 & $0.0510(15)$ & $0.0342(14)$ & $0.0335(13)$ & $0.0061(12)$ & $0.0093(12)$ & $0.0124(11)$ \\
O2 & $0.0398(14)$ & $0.0324(14)$ & $0.0416(14)$ & $0.0141(12)$ & $0.0061(12)$ & $0.0108(12)$ \\
C1 & $0.0287(18)$ & $0.0262(19)$ & $0.0240(17)$ & $-0.0001(14)$ & $-0.0020(14)$ & $-0.0015(14)$ \\
C2 & $0.0280(17)$ & $0.0190(16)$ & $0.0232(16)$ & $-0.0010(13)$ & $-0.0015(13)$ & $0.0000(13)$ \\
C3 & $0.0338(18)$ & $0.033(2)$ & $0.0291(18)$ & $0.0054(15)$ & $0.0141(15)$ & $0.0104(15)$ \\
C4 & $0.0319(18)$ & $0.0303(19)$ & $0.0329(18)$ & $0.0105(15)$ & $0.0142(15)$ & $0.0078(15)$ \\
C5 & $0.0235(16)$ & $0.0242(17)$ & $0.0206(16)$ & $-0.0005(13)$ & $0.0003(13)$ & $0.0012(13)$ \\
C6 & $0.0297(17)$ & $0.0253(18)$ & $0.0240(16)$ & $0.0025(14)$ & $0.0102(14)$ & $0.0048(14)$ \\
C7 & $0.0258(16)$ & $0.0241(17)$ & $0.0298(17)$ & $0.0061(13)$ & $0.0058(14)$ & $0.0042(14)$ \\
C8 & $0.045(2)$ & $0.0308(19)$ & $0.0326(19)$ & $0.0042(16)$ & $0.0076(16)$ & $0.0084(15)$ \\
C9 & $0.0279(18)$ & $0.0293(19)$ & $0.0386(19)$ & $-0.0012(14)$ & $0.0035(15)$ & $-0.0016(15)$ \\
C10 & $0.0236(17)$ & $0.039(2)$ & $0.046(2)$ & $0.0056(15)$ & $0.0030(15)$ & $0.0063(17)$ \\
Ge2 & $0.02168(19)$ & $0.0210(2)$ & $0.0313(2)$ & $0.00206(14)$ & $-0.00119(14)$ & $0.00386(15)$ \\
O3 & $0.0441(14)$ & $0.0212(12)$ & $0.0352(13)$ & $0.0003(11)$ & $-0.0028(11)$ & $0.0024(10)$ \\
O4 & $0.0411(15)$ & $0.0338(15)$ & $0.0379(14)$ & $0.0072(12)$ & $-0.0082(11)$ & $0.0060(12)$ \\
C11 & $0.0357(19)$ & $0.0264(19)$ & $0.0187(16)$ & $0.0078(15)$ & $0.0043(14)$ & $0.0010(14)$ \\
C12 & $0.0315(18)$ & $0.0261(18)$ & $0.0173(15)$ & $0.0040(14)$ & $0.0043(13)$ & $-0.0011(13)$ \\
C13 & $0.0284(17)$ & $0.0234(17)$ & $0.0302(18)$ & $-0.0018(14)$ & $0.0000(14)$ & $0.0020(14)$ \\
C14 & $0.0237(17)$ & $0.0269(18)$ & $0.0320(18)$ & $0.0025(14)$ & $-0.0028(14)$ & $0.0052(14)$ \\
C15 & $0.0257(17)$ & $0.0225(17)$ & $0.0250(17)$ & $0.0037(13)$ & $0.0019(13)$ & $0.0018(14)$ \\
C16 & $0.0299(18)$ & $0.0241(18)$ & $0.0363(19)$ & $-0.0045(14)$ & $-0.0038(15)$ & $0.0020(15)$ \\
C17 & $0.0250(17)$ & $0.0258(18)$ & $0.0321(18)$ & $0.0041(14)$ & $-0.0059(14)$ & $0.0030(14)$ \\
C18 & $0.035(2)$ & $0.040(2)$ & $0.044(2)$ & $0.0114(16)$ & $0.0014(17)$ & $-0.0034(17)$ \\
C19 & $0.0310(19)$ & $0.032(2)$ & $0.054(2)$ & $-0.0012(15)$ & $0.0039(17)$ & $0.0172(17)$ \\
C20 & $0.042(2)$ & $0.040(2)$ & $0.039(2)$ & $0.0076(17)$ & $-0.0100(17)$ & $0.0072(17)$ \\
& & & & & &
\end{tabular}


Geometric parameters $\left(\AA,{ }^{\circ}\right)$

\begin{tabular}{|c|c|c|c|}
\hline $\mathrm{Ge} 1-\mathrm{C} 5$ & $1.955(3)$ & $\mathrm{Ge} 2-\mathrm{C} 15$ & $1.955(3)$ \\
\hline $\mathrm{Ge} 1-\mathrm{C} 8$ & $1.942(3)$ & $\mathrm{Ge} 2-\mathrm{C} 18$ & $1.949(3)$ \\
\hline $\mathrm{Ge} 1-\mathrm{C} 9$ & $1.945(3)$ & $\mathrm{Ge} 2-\mathrm{C} 19$ & $1.938(3)$ \\
\hline $\mathrm{Ge} 1-\mathrm{C} 10$ & $1.939(3)$ & $\mathrm{Ge} 2-\mathrm{C} 20$ & $1.937(3)$ \\
\hline $\mathrm{O} 1-\mathrm{C} 1$ & $1.289(3)$ & $\mathrm{O} 3-\mathrm{C} 11$ & $1.250(4)$ \\
\hline $\mathrm{O} 2-\mathrm{H} 2$ & $0.93(5)$ & $\mathrm{O} 4-\mathrm{H} 4 \mathrm{~A}$ & $0.93(5)$ \\
\hline $\mathrm{O} 2-\mathrm{C} 1$ & $1.256(3)$ & $\mathrm{O} 4-\mathrm{C} 11$ & $1.295(4)$ \\
\hline $\mathrm{C} 1-\mathrm{C} 2$ & $1.476(4)$ & $\mathrm{C} 11-\mathrm{C} 12$ & $1.486(4)$ \\
\hline $\mathrm{C} 2-\mathrm{C} 3$ & $1.383(4)$ & $\mathrm{C} 12-\mathrm{C} 13$ & $1.385(4)$ \\
\hline $\mathrm{C} 2-\mathrm{C} 7$ & $1.384(4)$ & $\mathrm{C} 12-\mathrm{C} 17$ & $1.381(4)$ \\
\hline $\mathrm{C} 3-\mathrm{H} 3$ & 0.9500 & $\mathrm{C} 13-\mathrm{H} 13$ & 0.9500 \\
\hline $\mathrm{C} 3-\mathrm{C} 4$ & $1.368(4)$ & $\mathrm{C} 13-\mathrm{C} 14$ & $1.379(4)$ \\
\hline $\mathrm{C} 4-\mathrm{H} 4$ & 0.9500 & $\mathrm{C} 14-\mathrm{H} 14$ & 0.9500 \\
\hline $\mathrm{C} 4-\mathrm{C} 5$ & $1.393(4)$ & $\mathrm{C} 14-\mathrm{C} 15$ & $1.393(4)$ \\
\hline $\mathrm{C} 5-\mathrm{C} 6$ & $1.398(4)$ & $\mathrm{C} 15-\mathrm{C} 16$ & $1.398(4)$ \\
\hline $\mathrm{C} 6-\mathrm{H} 6$ & 0.9500 & C16-H16 & 0.9500 \\
\hline $\mathrm{C} 6-\mathrm{C} 7$ & $1.379(4)$ & $\mathrm{C} 16-\mathrm{C} 17$ & $1.374(4)$ \\
\hline $\mathrm{C} 7-\mathrm{H} 7$ & 0.9500 & C17-H17 & 0.9500 \\
\hline $\mathrm{C} 8-\mathrm{H} 8 \mathrm{~A}$ & 0.9800 & C18-H18A & 0.9800 \\
\hline $\mathrm{C} 8-\mathrm{H} 8 \mathrm{~B}$ & 0.9800 & C18-H18B & 0.9800 \\
\hline $\mathrm{C} 8-\mathrm{H} 8 \mathrm{C}$ & 0.9800 & $\mathrm{C} 18-\mathrm{H} 18 \mathrm{C}$ & 0.9800 \\
\hline C9-H9A & 0.9800 & C19-H19A & 0.9800 \\
\hline C9-H9B & 0.9800 & C19-H19B & 0.9800 \\
\hline $\mathrm{C} 9-\mathrm{H} 9 \mathrm{C}$ & 0.9800 & C19-H19C & 0.9800 \\
\hline $\mathrm{C} 10-\mathrm{H} 10 \mathrm{~A}$ & 0.9800 & $\mathrm{C} 20-\mathrm{H} 20 \mathrm{~A}$ & 0.9800 \\
\hline $\mathrm{C} 10-\mathrm{H} 10 \mathrm{~B}$ & 0.9800 & $\mathrm{C} 20-\mathrm{H} 20 \mathrm{~B}$ & 0.9800 \\
\hline $\mathrm{C} 10-\mathrm{H} 10 \mathrm{C}$ & 0.9800 & $\mathrm{C} 20-\mathrm{H} 20 \mathrm{C}$ & 0.9800 \\
\hline $\mathrm{C} 8-\mathrm{Ge} 1-\mathrm{C} 5$ & $109.93(12)$ & $\mathrm{C} 18-\mathrm{Ge} 2-\mathrm{C} 15$ & $108.31(13)$ \\
\hline $\mathrm{C} 8-\mathrm{Ge} 1-\mathrm{C} 9$ & $109.99(13)$ & $\mathrm{C} 19-\mathrm{Ge} 2-\mathrm{C} 15$ & $108.52(13)$ \\
\hline $\mathrm{C} 9-\mathrm{Ge} 1-\mathrm{C} 5$ & $107.50(13)$ & $\mathrm{C} 19-\mathrm{Ge} 2-\mathrm{C} 18$ & $110.01(15)$ \\
\hline $\mathrm{C} 10-\mathrm{Ge} 1-\mathrm{C} 5$ & $109.30(13)$ & $\mathrm{C} 20-\mathrm{Ge} 2-\mathrm{C} 15$ & $108.88(13)$ \\
\hline $\mathrm{C} 10-\mathrm{Ge} 1-\mathrm{C} 8$ & $109.89(14)$ & $\mathrm{C} 20-\mathrm{Ge} 2-\mathrm{C} 18$ & $109.23(14)$ \\
\hline $\mathrm{C} 10-\mathrm{Ge} 1-\mathrm{C} 9$ & $110.19(13)$ & $\mathrm{C} 20-\mathrm{Ge} 2-\mathrm{C} 19$ & $111.81(15)$ \\
\hline $\mathrm{C} 1-\mathrm{O} 2-\mathrm{H} 2$ & $122(3)$ & $\mathrm{C} 11-\mathrm{O} 4-\mathrm{H} 4 \mathrm{~A}$ & $116(3)$ \\
\hline $\mathrm{O} 1-\mathrm{C} 1-\mathrm{C} 2$ & $117.5(3)$ & $\mathrm{O} 3-\mathrm{C} 11-\mathrm{O} 4$ & $123.6(3)$ \\
\hline $\mathrm{O} 2-\mathrm{C} 1-\mathrm{O} 1$ & $123.2(3)$ & $\mathrm{O} 3-\mathrm{C} 11-\mathrm{C} 12$ & $120.3(3)$ \\
\hline $\mathrm{O} 2-\mathrm{C} 1-\mathrm{C} 2$ & $119.3(3)$ & $\mathrm{O} 4-\mathrm{C} 11-\mathrm{C} 12$ & $116.0(3)$ \\
\hline $\mathrm{C} 3-\mathrm{C} 2-\mathrm{C} 1$ & $120.8(3)$ & $\mathrm{C} 13-\mathrm{C} 12-\mathrm{C} 11$ & $120.0(3)$ \\
\hline $\mathrm{C} 3-\mathrm{C} 2-\mathrm{C} 7$ & $118.6(3)$ & $\mathrm{C} 17-\mathrm{C} 12-\mathrm{C} 11$ & $120.6(3)$ \\
\hline $\mathrm{C} 7-\mathrm{C} 2-\mathrm{C} 1$ & $120.6(3)$ & $\mathrm{C} 17-\mathrm{C} 12-\mathrm{C} 13$ & $119.3(3)$ \\
\hline $\mathrm{C} 2-\mathrm{C} 3-\mathrm{H} 3$ & 119.6 & $\mathrm{C} 12-\mathrm{C} 13-\mathrm{H} 13$ & 120.1 \\
\hline $\mathrm{C} 4-\mathrm{C} 3-\mathrm{C} 2$ & $120.8(3)$ & $\mathrm{C} 14-\mathrm{C} 13-\mathrm{C} 12$ & $119.7(3)$ \\
\hline $\mathrm{C} 4-\mathrm{C} 3-\mathrm{H} 3$ & 119.6 & $\mathrm{C} 14-\mathrm{C} 13-\mathrm{H} 13$ & 120.1 \\
\hline $\mathrm{C} 3-\mathrm{C} 4-\mathrm{H} 4$ & 119.1 & $\mathrm{C} 13-\mathrm{C} 14-\mathrm{H} 14$ & 119.0 \\
\hline $\mathrm{C} 3-\mathrm{C} 4-\mathrm{C} 5$ & $121.7(3)$ & $\mathrm{C} 13-\mathrm{C} 14-\mathrm{C} 15$ & $122.0(3)$ \\
\hline
\end{tabular}




\begin{tabular}{|c|c|c|c|}
\hline $\mathrm{C} 5-\mathrm{C} 4-\mathrm{H} 4$ & 119.1 & $\mathrm{C} 15-\mathrm{C} 14-\mathrm{H} 14$ & 119.0 \\
\hline $\mathrm{C} 4-\mathrm{C} 5-\mathrm{Ge} 1$ & $121.8(2)$ & $\mathrm{C} 14-\mathrm{C} 15-\mathrm{Ge} 2$ & $122.6(2)$ \\
\hline $\mathrm{C} 4-\mathrm{C} 5-\mathrm{C} 6$ & $116.8(3)$ & $\mathrm{C} 14-\mathrm{C} 15-\mathrm{C} 16$ & $117.0(3)$ \\
\hline $\mathrm{C} 6-\mathrm{C} 5-\mathrm{Ge} 1$ & $121.2(2)$ & $\mathrm{C} 16-\mathrm{C} 15-\mathrm{Ge} 2$ & $120.4(2)$ \\
\hline $\mathrm{C} 5-\mathrm{C} 6-\mathrm{H} 6$ & 119.3 & $\mathrm{C} 15-\mathrm{C} 16-\mathrm{H} 16$ & 119.3 \\
\hline $\mathrm{C} 7-\mathrm{C} 6-\mathrm{C} 5$ & $121.5(3)$ & $\mathrm{C} 17-\mathrm{C} 16-\mathrm{C} 15$ & $121.4(3)$ \\
\hline $\mathrm{C} 7-\mathrm{C} 6-\mathrm{H} 6$ & 119.3 & $\mathrm{C} 17-\mathrm{C} 16-\mathrm{H} 16$ & 119.3 \\
\hline $\mathrm{C} 2-\mathrm{C} 7-\mathrm{H} 7$ & 119.8 & $\mathrm{C} 12-\mathrm{C} 17-\mathrm{H} 17$ & 119.7 \\
\hline $\mathrm{C} 6-\mathrm{C} 7-\mathrm{C} 2$ & $120.4(3)$ & $\mathrm{C} 16-\mathrm{C} 17-\mathrm{C} 12$ & $120.6(3)$ \\
\hline $\mathrm{C} 6-\mathrm{C} 7-\mathrm{H} 7$ & 119.8 & $\mathrm{C} 16-\mathrm{C} 17-\mathrm{H} 17$ & 119.7 \\
\hline $\mathrm{Ge} 1-\mathrm{C} 8-\mathrm{H} 8 \mathrm{~A}$ & 109.5 & $\mathrm{Ge} 2-\mathrm{C} 18-\mathrm{H} 18 \mathrm{~A}$ & 109.5 \\
\hline $\mathrm{Ge} 1-\mathrm{C} 8-\mathrm{H} 8 \mathrm{~B}$ & 109.5 & $\mathrm{Ge} 2-\mathrm{C} 18-\mathrm{H} 18 \mathrm{~B}$ & 109.5 \\
\hline $\mathrm{Ge} 1-\mathrm{C} 8-\mathrm{H} 8 \mathrm{C}$ & 109.5 & $\mathrm{Ge} 2-\mathrm{C} 18-\mathrm{H} 18 \mathrm{C}$ & 109.5 \\
\hline $\mathrm{H} 8 \mathrm{~A}-\mathrm{C} 8-\mathrm{H} 8 \mathrm{~B}$ & 109.5 & $\mathrm{H} 18 \mathrm{~A}-\mathrm{C} 18-\mathrm{H} 18 \mathrm{~B}$ & 109.5 \\
\hline $\mathrm{H} 8 \mathrm{~A}-\mathrm{C} 8-\mathrm{H} 8 \mathrm{C}$ & 109.5 & $\mathrm{H} 18 \mathrm{~A}-\mathrm{C} 18-\mathrm{H} 18 \mathrm{C}$ & 109.5 \\
\hline $\mathrm{H} 8 \mathrm{~B}-\mathrm{C} 8-\mathrm{H} 8 \mathrm{C}$ & 109.5 & $\mathrm{H} 18 \mathrm{~B}-\mathrm{C} 18-\mathrm{H} 18 \mathrm{C}$ & 109.5 \\
\hline $\mathrm{Ge} 1-\mathrm{C} 9-\mathrm{H} 9 \mathrm{~A}$ & 109.5 & $\mathrm{Ge} 2-\mathrm{C} 19-\mathrm{H} 19 \mathrm{~A}$ & 109.5 \\
\hline $\mathrm{Ge} 1-\mathrm{C} 9-\mathrm{H} 9 \mathrm{~B}$ & 109.5 & $\mathrm{Ge} 2-\mathrm{C} 19-\mathrm{H} 19 \mathrm{~B}$ & 109.5 \\
\hline $\mathrm{Ge} 1-\mathrm{C} 9-\mathrm{H} 9 \mathrm{C}$ & 109.5 & $\mathrm{Ge} 2-\mathrm{C} 19-\mathrm{H} 19 \mathrm{C}$ & 109.5 \\
\hline $\mathrm{H} 9 \mathrm{~A}-\mathrm{C} 9-\mathrm{H} 9 \mathrm{~B}$ & 109.5 & $\mathrm{H} 19 \mathrm{~A}-\mathrm{C} 19-\mathrm{H} 19 \mathrm{~B}$ & 109.5 \\
\hline $\mathrm{H} 9 \mathrm{~A}-\mathrm{C} 9-\mathrm{H} 9 \mathrm{C}$ & 109.5 & $\mathrm{H} 19 \mathrm{~A}-\mathrm{C} 19-\mathrm{H} 19 \mathrm{C}$ & 109.5 \\
\hline $\mathrm{H} 9 \mathrm{~B}-\mathrm{C} 9-\mathrm{H} 9 \mathrm{C}$ & 109.5 & $\mathrm{H} 19 \mathrm{~B}-\mathrm{C} 19-\mathrm{H} 19 \mathrm{C}$ & 109.5 \\
\hline $\mathrm{Ge} 1-\mathrm{C} 10-\mathrm{H} 10 \mathrm{~A}$ & 109.5 & $\mathrm{Ge} 2-\mathrm{C} 20-\mathrm{H} 20 \mathrm{~A}$ & 109.5 \\
\hline $\mathrm{Ge} 1-\mathrm{C} 10-\mathrm{H} 10 \mathrm{~B}$ & 109.5 & $\mathrm{Ge} 2-\mathrm{C} 20-\mathrm{H} 20 \mathrm{~B}$ & 109.5 \\
\hline $\mathrm{Ge} 1-\mathrm{C} 10-\mathrm{H} 10 \mathrm{C}$ & 109.5 & $\mathrm{Ge} 2-\mathrm{C} 20-\mathrm{H} 20 \mathrm{C}$ & 109.5 \\
\hline $\mathrm{H} 10 \mathrm{~A}-\mathrm{C} 10-\mathrm{H} 10 \mathrm{~B}$ & 109.5 & $\mathrm{H} 20 \mathrm{~A}-\mathrm{C} 20-\mathrm{H} 20 \mathrm{~B}$ & 109.5 \\
\hline $\mathrm{H} 10 \mathrm{~A}-\mathrm{C} 10-\mathrm{H} 10 \mathrm{C}$ & 109.5 & $\mathrm{H} 20 \mathrm{~A}-\mathrm{C} 20-\mathrm{H} 20 \mathrm{C}$ & 109.5 \\
\hline $\mathrm{H} 10 \mathrm{~B}-\mathrm{C} 10-\mathrm{H} 10 \mathrm{C}$ & 109.5 & $\mathrm{H} 20 \mathrm{~B}-\mathrm{C} 20-\mathrm{H} 20 \mathrm{C}$ & 109.5 \\
\hline $\mathrm{Ge} 1-\mathrm{C} 5-\mathrm{C} 6-\mathrm{C} 7$ & $-173.3(2)$ & $\mathrm{Ge} 2-\mathrm{C} 15-\mathrm{C} 16-\mathrm{C} 17$ & $176.5(2)$ \\
\hline $\mathrm{O} 1-\mathrm{C} 1-\mathrm{C} 2-\mathrm{C} 3$ & $3.4(4)$ & $\mathrm{O} 3-\mathrm{C} 11-\mathrm{C} 12-\mathrm{C} 13$ & $4.5(4)$ \\
\hline $\mathrm{O} 1-\mathrm{C} 1-\mathrm{C} 2-\mathrm{C} 7$ & $-175.0(3)$ & $\mathrm{O} 3-\mathrm{C} 11-\mathrm{C} 12-\mathrm{C} 17$ & $-175.6(3)$ \\
\hline $\mathrm{O} 2-\mathrm{C} 1-\mathrm{C} 2-\mathrm{C} 3$ & $-176.0(3)$ & $\mathrm{O} 4-\mathrm{C} 11-\mathrm{C} 12-\mathrm{C} 13$ & $-175.5(3)$ \\
\hline $\mathrm{O} 2-\mathrm{C} 1-\mathrm{C} 2-\mathrm{C} 7$ & $5.6(5)$ & $\mathrm{O} 4-\mathrm{C} 11-\mathrm{C} 12-\mathrm{C} 17$ & $4.4(4)$ \\
\hline $\mathrm{C} 1-\mathrm{C} 2-\mathrm{C} 3-\mathrm{C} 4$ & $-176.4(3)$ & $\mathrm{C} 11-\mathrm{C} 12-\mathrm{C} 13-\mathrm{C} 14$ & $179.3(3)$ \\
\hline $\mathrm{C} 1-\mathrm{C} 2-\mathrm{C} 7-\mathrm{C} 6$ & $176.4(3)$ & $\mathrm{C} 11-\mathrm{C} 12-\mathrm{C} 17-\mathrm{C} 16$ & $-179.6(3)$ \\
\hline $\mathrm{C} 2-\mathrm{C} 3-\mathrm{C} 4-\mathrm{C} 5$ & $0.1(5)$ & $\mathrm{C} 12-\mathrm{C} 13-\mathrm{C} 14-\mathrm{C} 15$ & $-0.1(5)$ \\
\hline $\mathrm{C} 3-\mathrm{C} 2-\mathrm{C} 7-\mathrm{C} 6$ & $-2.0(5)$ & $\mathrm{C} 13-\mathrm{C} 12-\mathrm{C} 17-\mathrm{C} 16$ & $0.3(4)$ \\
\hline $\mathrm{C} 3-\mathrm{C} 4-\mathrm{C} 5-\mathrm{Ge} 1$ & $173.2(2)$ & $\mathrm{C} 13-\mathrm{C} 14-\mathrm{C} 15-\mathrm{Ge} 2$ & $-176.8(2)$ \\
\hline $\mathrm{C} 3-\mathrm{C} 4-\mathrm{C} 5-\mathrm{C} 6$ & $-2.1(5)$ & $\mathrm{C} 13-\mathrm{C} 14-\mathrm{C} 15-\mathrm{C} 16$ & $1.0(4)$ \\
\hline $\mathrm{C} 4-\mathrm{C} 5-\mathrm{C} 6-\mathrm{C} 7$ & $2.1(4)$ & $\mathrm{C} 14-\mathrm{C} 15-\mathrm{C} 16-\mathrm{C} 17$ & $-1.3(4)$ \\
\hline $\mathrm{C} 5-\mathrm{C} 6-\mathrm{C} 7-\mathrm{C} 2$ & $0.0(5)$ & $\mathrm{C} 15-\mathrm{C} 16-\mathrm{C} 17-\mathrm{C} 12$ & $0.7(5)$ \\
\hline $\mathrm{C} 7-\mathrm{C} 2-\mathrm{C} 3-\mathrm{C} 4$ & $2.0(5)$ & $\mathrm{C} 17-\mathrm{C} 12-\mathrm{C} 13-\mathrm{C} 14$ & $-0.6(4)$ \\
\hline
\end{tabular}

Hydrogen-bond geometry $\left(\AA,{ }^{\circ}\right)$

\begin{tabular}{lllll}
\hline$D-\mathrm{H} \cdots A$ & $D-\mathrm{H}$ & $\mathrm{H} \cdots A$ & $D \cdots A$ & $D-\mathrm{H} \cdots A$ \\
\hline $\mathrm{O} 2-\mathrm{H} 2 \cdots \mathrm{O} 1^{\mathrm{i}}$ & $0.93(5)$ & $1.71(5)$ & $2.626(3)$ & $170(5)$
\end{tabular}


$\mathrm{O} 4-\mathrm{H} 4 A \cdots \mathrm{O} 3^{\mathrm{ii}}$

$0.93(5)$

$1.70(5)$

$2.635(3)$

$179(4)$

Symmetry codes: (i) $-x,-y+2,-z$; (ii) $-x+2,-y+1,-z+1$. 